\title{
CHIANTI-AN ATOMIC DATABASE FOR EMISSION LINES. XII. VERSION 7 OF THE DATABASE
}

\author{
E. Landi ${ }^{1}$, G. Del Zanna ${ }^{2}$, P. R. Young ${ }^{3}$, K. P. Dere ${ }^{4}$, and H. E. Mason ${ }^{2}$ \\ ${ }^{1}$ Department of Atmospheric, Oceanic and Space Sciences, University of Michigan, Ann Arbor, MI 48109, USA \\ ${ }^{2}$ Department of Applied Mathematics and Theoretical Physics, University of Cambridge, Wilberforce Road, Cambridge CB3 0WA, UK \\ ${ }^{3}$ College of Science, George Mason University, 4400 University Drive, Fairfax, VA, 22030, USA \\ ${ }^{4}$ School of Physics, Astronomy and Computational Sciences, MS 6A2, George Mason University, 4400 University Drive, Fairfax, VA 22030, USA \\ Received 2011 July 13; accepted 2011 September 13; published 2011 December 19
}

\begin{abstract}
The CHIANTI spectral code consists of an atomic database and a suite of computer programs to calculate the optically thin spectrum of astrophysical objects and carry out spectroscopic plasma diagnostics. The database includes atomic energy levels, wavelengths, radiative transition probabilities, collision excitation rate coefficients, and ionization and recombination rate coefficients, as well as data to calculate free-free, free-bound, and two-photon continuum emission. Version 7 has been released, which includes several new ions, significant updates to existing ions, as well as Chianti-Py, the implementation of CHIANTI software in the Python programming language. All data and programs are freely available at http://www.chiantidatabase.org, while the Python interface to CHIANTI can be found at http://chiantipy.sourceforge.net.
\end{abstract}

Key words: atomic data - atomic processes - Sun: UV radiation - Sun: X-rays, gamma rays - ultraviolet: general - X-rays: general

\section{INTRODUCTION}

CHIANTI is a database of assessed atomic parameters and transition rates necessary for the calculation of the emissivity of optically thin, collisionally dominated plasmas. IDL-based software was initially developed to allow for synthetic spectra calculation and plasma diagnostics. CHIANTI was first released in 1996 (Dere et al. 1997) and it covered wavelengths larger than $50 \AA$. Since its first release, several updates have been made to both the database and the software and released to the astrophysical community, aimed at (1) expanding the database, (2) improving the quality of the CHIANTI data, (3) including new physical processes, and (4) facilitating the use of CHIANTI data and software in major solar missions such as the Solar and Heliospheric Observatory (SOHO), Transition Region and Coronal Explorer (TRACE), Reuven Ramaty High Energy Solar Spectroscopic Imager (RHESSI), Solar Terrestial Relations Observatory (STEREO), Hinode, and the Solar Dynamics Observatory (SDO). Also, several benchmark studies have been carried out by members of the CHIANTI team to ensure the quality of the CHIANTI data and identify areas where improvement was needed. As a result of the efforts of the CHIANTI team, CHIANTI is one of the most widespread, complete, and accurate spectral codes available for optically thin emission in the 1-2000 A wavelength range.

Since the latest release (Dere et al. 2009), many new atomic physics calculations were published that improved the data sets for several ions in CHIANTI, or provided new data for transitions unavailable in the literature before. Also, a new suite of codes written in the Python computer language has been developed and released, which also allows the use of CHIANTI data for plasma diagnostics and synthetic spectra calculation. The present paper describes the latest version of the CHIANTI database (version 7.0), which has been developed to include both the new calculations and the Python software. The CHIANTI database includes several files for each ion: the information on the atomic model and energy level is stored in the "ELVLC" file (for example, si_9.elvlc for Si IX); wavelengths and radiative data are stored in the "WGFA" file (e.g., si_9.wg $f a$ for Si IX), and we will use the ELVLC and WGFA conventions throughout the text.

\section{DATABASE IMPROVEMENTS}

\subsection{H-like Ions}

\subsubsection{Ar XVIII}

The new data for Ar XVIII replace the entire set of CHIANTI files, which were based on interpolation of $R$-matrix calculations available in CHIANTI for the other members of the H-like sequence. Version 7 includes the large-scale calculation of Aggarwal et al. (2008), which provides a complete set of data for all the configurations up to $n=5$, for a total of 25 fine-structure levels. Experimental energy levels for the $2 p^{2} P$ doublet come from solar flare observations by Phillips et al. (2003), while the rest of the data are taken from version 3 of the NIST database (Ralchenko et al. 2008).

Wave functions, energy levels, and $A$ values for electric and magnetic dipole and quadrupole transitions were calculated by Aggarwal et al. (2008) using an updated version of the GRASP code (Grant et al. 1980) for all transition in the atomic model. The magnetic dipole and two-photon decay rates from the metastable $2 s{ }^{2} S_{1 / 2}$ level from Parpia \& Johnson (1982) are retained. Collision strengths have been calculated using an updated version of the Dirac Atomic R-Matrix Code (DARC) program (made available to the authors by Norrington and Grant) including resonant excitation. Maxwellian-averaged collision strengths were calculated by Aggarwal et al. (2008) in the $5.6 \leqslant \log T \leqslant 7.4$ temperature range. The Aggarwal et al. (2008) data change the predicted intensities of the X-ray lines over the previous CHIANTI version by up to $20 \%$, consistently with the differences in the atomic data for those transitions; for other transitions, larger differences were found and the Aggarwal et al. (2008) data represent a significant improvement over the interpolated values. The ratio between the two components of the Ly $\alpha$ doublet at $3.73 \AA$ was recently measured by Rice et al. (2011) to be $0.524 \pm 0.006$, which 
compares more favorably with the CHIANTI version 7 value of 0.517 rather than with the previous value of 0.501 .

\subsection{He-like Ions}

\subsection{1. $N V I$}

The data for NVI in the previous version of CHIANTI consisted of a mixture of $A$ values from a variety of sources, and of extrapolated effective collision strengths. The latter were obtained using the available data for O VII, Ne IX, and Mg XI from Zhang \& Sampson (1987). We have replaced this data set entirely with the calculations by Aggarwal et al. (2009), who provide theoretical energy levels, $A$ values, and effective collision strengths, only retaining from the previous CHIANTI model the experimental values of the energy levels (from NIST 1.0-Martin et al. 1995; Chen et al. 1993), the laboratory $A$ values (measured by Neill et al. 2000 and Hutton et al. 1985), and the two-photon transition rate from Drake (1986).

The atomic model of Aggarwal et al. (2009) includes all the configurations up to $n=5$ for a total of 49 fine-structure levels, all included in CHIANTI. Following the recommendations of Aggarwal et al. (2009), we included theoretical energy levels and A values from their "FAC1" calculation, carried out using the Flexible Atomic Code (FAC;- Gu 2003) on an atomic model which includes up to the $n=5$ configuration. Theoretical energy levels agree with NIST values to better than $0.25 \%$, while the calculated $A$ values agree with the Neill et al. (2000) and Hutton et al. (1985) to better than 12\%. Collision strengths were calculated using the DARC including the contribution of resonances, and averaged over a Maxwellian distribution in the temperature range $4.0 \leqslant \log T \leqslant 6.5$. Line intensities for the observed lines calculated with Aggarwal et al. (2009) agree with CHIANTI 6 values within $30 \%$, consistently with the differences in the atomic data; for the other transitions, larger differences are found and the Aggarwal et al. (2009) data represent a significant improvement over the interpolated values.

\subsection{2. $\mathrm{NaX}$}

The data for $\mathrm{Nax}$ have been completely revised. The old versions of CHIANTI included a data set that was obtained through interpolation of data available throughout the entire He-like sequence. In the present version we include the largescale calculation of Aggarwal et al. (2009). Experimental energy levels are taken from the version 3 of NIST (Ralchenko et al. 2008) with the exception of the $1 s 2 p^{3} P_{2}$ level, taken from solar flare observations in the ultraviolet made by Curdt et al. (2000), who provide a more accurate estimate.

The calculations are overall similar to those made for $\mathrm{N}$ VI; we used the energy levels and $A$ values of the FAC 1 calculations recommended by Aggarwal et al. (2009), who also provided Maxwellian-averaged collision strengths in the $5.0 \leqslant \log T \leqslant$ 7.0. The new set of data changes line emissivities by $5 \%-10 \%$ for the transitions within the $n=2$ configurations, and by up to $30 \%$ for transitions connecting the $n=2,3$, and 4 configurations. Comparison between the Aggarwal et al. (2009) and the interpolated values in CHIANTI 6 provides similar results as for $\mathrm{N}$ VI.

\subsubsection{ArXVII}

Most of the data for Ar XVII have been updated using the calculations of Aggarwal \& Keenan (2005). We also updated the experimental energies of this ion, and from the previous version of CHIANTI we only retained the collisional data for the transitions involving levels in the $n=2,3$ and $n=4$ configurations, from Whiteford et al. (2001), since Whiteford et al. (2001) take into account the effect of radiation damping of resonances, neglected by Aggarwal \& Keenan (2005).

Experimental energy levels come from NIST version 3 (Ralchenko et al. 2008), with the exception of the levels in the $n=2$ configuration (taken from a combination of measurements from Neupert 1971, Walker et al. 1974, Dohmann \& Pfeng 1978, Dohmann et al. 1978, Dohmann \& Mann 1979, Briand et al. 1983, Beyer et al. 1986, and Sylwester et al. 2003) and level $1 s 3{ }^{1} P_{1}$, which comes from Neupert (1971).

Aggarwal \& Keenan (2005) provide $A$ values and effective collision strengths for all transitions between the 49 finestructure levels from all configurations with $n \leqslant 5$ included in their atomic model. Their $A$ values replace those in previous versions of CHIANTI, except the two-photon decay rate from Drake (1986), which they do not calculate. Effective collision strengths are provided by Aggarwal \& Keenan (2005) in the $5.2 \leqslant \log T \leqslant 7.2$ temperature range.

\subsection{Be-like Ions \\ 2.3.1. $M g I X$}

Del Zanna et al. (2008) performed an Intermediate Coupling Frame Transformation (ICFT; described in Griffin et al. 1998) R-matrix calculation for $\mathrm{Mg}$ IX including a total of 98 finestructure levels, up to $n=4$. Previously, Keenan et al. (1986) published a tabulation of interpolated electron excitation rates for some ions along the Be-like sequence for the $n=2 \rightarrow 2$ transitions. The Del Zanna et al. (2008) results are, for some lines, significantly different than the interpolated values. Del Zanna et al. (2008) calculated transition probabilities for all of the 4753 transitions with AUTOSTRUCTURE (Badnell 1997), adopting experimental energies whenever available. The excitation and radiative rates of Del Zanna et al. (2008) are adopted here, and replace the Keenan et al. (1986) $n=2$ data and the Bhatia \& Landi (2007) $n=3,4$ distorted wave (DW) data. Fits to the original effective collision strengths are accurate (to within $\approx 1 \%$ ) in the $5.2 \leqslant \log T \leqslant 6.8$ temperature range. The experimental energy levels come from the NIST database. The Bhatia \& Landi (2007) $n=5$ data and experimental energy levels have been retained by changing the indices. Previous long-standing discrepancies in solar coronal temperatures measured with Mg IX are now resolved (see Del Zanna et al. 2008).

\subsection{B-like Ions}

$$
\text { 2.4.1. Si } X
$$

Liang et al. (2009) has produced a large ICFT R-matrix calculation including 125 fine-structure levels (58 LS terms) belonging to the spectroscopically important $n=2,3$ configurations and we use them in this version of CHIANTI. The new excitation rates result in significantly increased emissivity in many X-ray and EUV lines from previous calculations. Liang et al. (2009) also provided oscillator strengths and $A$ values. We have fitted the Liang et al. effective collision strengths with nine-point spline fits, taking into account the high-energy limits. The fits are accurate (to within $\approx 1 \%$ ) between $10^{5} \mathrm{~K}$ and $10^{7} \mathrm{~K}$.

All the observed energies for this ion have been reassessed using the original wavelength observations. The accurate energy of the ground term splitting is from Penn \& Kuhn (1994). The energies of the $2 s 2 p^{2}{ }^{4} P$ levels are from $\mathrm{SOHO} / \mathrm{SUMER}$ measurements (see Feldman \& Doschek 2007). The rest of the 
energies have been obtained from wavelength measurements, in particular from the very accurate wavelengths by Ferner (1941, 1948), Fawcett (1970), Hoory et al. (1971), and Behring et al. (1976). Many Si x line identifications were revised by Vilkas et al. (2005) using beam-foil spectra, and in most cases their suggestions have been adopted here. Note that for many levels NIST only assigned relative energies between levels from the same multiplet. The ambiguities have now been resolved.

\subsection{C-like Ions}

\subsubsection{Si IX}

The energy of the metastable level $2 s 2 p^{3}{ }^{5} S_{2}$ has been changed so that the CHIANTI wavelengths of the $2 s^{2} 2 p^{2}$ ${ }^{3} P_{1,2}-2 s 2 p^{3}{ }^{5} S_{2}$ transitions could match the values observed by the $S O H O / S U M E R$ instrument (Wilhelm et al. 1995) at $\lambda 676.51$ and $\lambda 694.69$. The previous value of the ${ }^{5} S_{2}$ energy came from Edlén (1985) and provided wavelengths larger than observed by $0.2 \AA$. The ${ }^{5} S_{2}$ energy was determined from the wavelength measured by Curdt et al. (2004), using the energies of the $2 s^{2} 2 p^{2}{ }^{3} P_{1,2}$ level available in CHIANTI (from Edlén 1985), and found $E_{5} S_{2}=150,364 \pm 4 \mathrm{~cm}^{-1}$, estimating the uncertainty by conservatively associating an uncertainty of $\Delta \lambda=0.02 \AA$ to the SUMER wavelengths. This value needs to be compared with Edlén's value $E_{5} S_{2}=150,320 \mathrm{~cm}^{-1}$. It is important to note that the energy separation $\Delta E=3868.4 \pm 8.5 \mathrm{~cm}^{-1}$ between the ${ }^{3} P_{1,2}$ levels in the ground configuration measured from the Curdt et al. (2004) wavelengths is in excellent agreement with $\Delta E=3869 \mathrm{~cm}^{-1}$ from Edlén (1985) available in CHIANTI.

\subsection{N-like Ions}

\subsubsection{Ne IV}

The atomic model for Ne IV in CHIANTI 6 is the same as that first introduced in CHIANTI 2 (Landi et al. 1999), and is not ideal for the forbidden transitions. First, the collision data of Ramsbottom et al. (1998) were truncated to temperatures $\log T \geqslant 4.4$ as it was not possible to fit the complete range of data using the five-point spline fits then used for CHIANTI. Since that work, nine-point spline fits have been implemented in the CHIANTI software and so the ground configuration transitions have been re-fitted. The fits now reproduce the original data to within $0.90 \%$ over the temperature range $4.0 \times 10^{3}$ to $7.9 \times 10^{5} \mathrm{~K}$. The radiative decay rates in the previous CHIANTI model were from an unpublished calculation by the CHIANTI team (Landi et al. 1999) carried out with SUPERSTRUCTURE (Eissner et al. 1974), and the forbidden transition rates have now been replaced with the values from Merkelis et al. (1999). Differences between the old CHIANTI $A$ values and the Merkelis et al. (1999) ones are within $30 \%$, with only one exception $\left({ }^{2} D_{3 / 2}-{ }^{2} P_{3 / 2}\right)$ having a difference of $40 \%$. In addition the Ne IV experimental energy values have been replaced with the values from Kramida et al. (1999).

\subsection{2. $\mathrm{NaV}$}

The electron collision strengths and radiative data for $\mathrm{Na} \mathrm{V}$ in earlier versions of CHIANTI were derived through interpolation along the isoelectronic sequence, while energy levels were taken from Edlén (1984). The model consisted of the 13 levels from the $2 s^{2} 2 p^{3}$ and $2 s 2 p^{4}$ configurations.

For CHIANTI 7, the radiative data have been replaced with those calculated by Merkelis et al. (1997) and Merkelis et al. (1999). The new decay rates for the strong allowed transitions are around $30 \%$ lower than the interpolated values, while the forbidden transition decay rates are higher by factors of up to seven.

\subsection{O-like Ions}

2.7.1. $O_{I}$

$\mathrm{O} \mathrm{I}$ is a new addition to CHIANTI and the model consists of the seven energetically lowest levels, $2 s^{2} 2 p^{4}{ }^{3} P_{J},{ }^{1} D_{2},{ }^{1} S_{0}$, and $2 s^{2} 2 p^{3} 3 s{ }^{5} S_{2},{ }^{3} S_{1}$. Energy levels are obtained from version 3 of the NIST database, and radiative decay rates are from Froese Fischer \& Tachiev (2004). The fine-structure effective collision strengths for transitions between the ${ }^{3} P_{J}$ levels are from Bell et al. (1998) and for all other transitions are from Zatsarinny $\&$ Tayal (2003). The latter work only gave collision strengths between LS terms, so the transitions between the $2 s^{2} 2 p^{4}{ }^{1} D_{2}$, ${ }^{1} S_{0}, 2 s^{2} 2 p^{3} 3 s^{5} S_{2},{ }^{3} S_{1}$ levels and the ground term have been split according to the statistical weights of the ${ }^{3} P$ levels.

The Bell et al. (1998) collision data are tabulated for seven temperatures from $50 \mathrm{~K}$ to $3000 \mathrm{~K}$, and the CHIANTI fits reproduce the original data to within $3.2 \%$ over this range. The Zatsarinny \& Tayal (2003) data are tabulated for 20 temperatures between 1000 and 60,000 K and the CHIANTI fits reproduce the original data to within $0.9 \%$ over this range.

\subsection{Ne-like Ions}

\subsubsection{Fe XVII}

A new R-matrix scattering calculation for all Ne-like ions from $\mathrm{Na}^{+}$to $\mathrm{Kr}^{26+}$ has been published by Liang \& Badnell (2010), and we use it in CHIANTI 7. Liang \& Badnell (2010) used the ICFT approach, with a close-coupling expansion including the 113 LS terms (209 levels) belonging to the configurations $2 s^{2} 2 p^{6}, 2 s^{2} 2 p^{5}(3,4,5) l, 2 s 2 p^{6}(3,4,5) l$ $(l=s, p, d, f, g)$, and $2 s^{2} 2 p^{5}(6,7) l(l=s, p, d)$. A larger configuration-interaction effect from additional configurations $\left(2 s^{2} 2 p^{4} 3 l(3,4,5) l^{\prime}\right.$ with $\left.l^{\prime}=s, p, d, f, g\right)$ was included in the target expansion. Liang \& Badnell (2010) also provide oscillator strengths and $A$ values, which replace the previous CHIANTI values.

The Liang \& Badnell R-matrix calculations are an improvement over the previous Landi \& Gu (2006) DW calculations because they include a proper treatment of the resonances, which are known to be important. The Liang \& Badnell calculations also improve the R-matrix results of Loch et al. (2006) because of the larger target adopted, although the collision strengths were similar, to within $20 \%$, as described in their paper. Del Zanna (2011a) presents a benchmark of these three atomic data sets against astrophysical $\mathrm{X}$-ray observations and confirms an overall agreement (within $\approx 10 \%$ ) in the predicted intensities of the strongest lines, with the Liang \& Badnell data sets agreeing better with observations. The Liang \& Badnell data provide instead significantly different EUV emissivities compared to the previous ones, as discussed in the benchmark work of Del Zanna \& Ishikawa (2009).

The Del Zanna \& Ishikawa (2009) identifications and energies (based on Hinode/EIS observations) have been adopted here. We have included $n=6,7$ observed energies obtained from the Brown et al. (1998) observed wavelengths.

We have fitted the Liang \& Badnell effective collision strengths with nine-point spline fits, taking into account the high-energy limits. The fits are accurate (to within $\approx 1 \%$ ) in the $10^{5}-10^{8} \mathrm{~K}$ range. The Landi \& Gu (2006) DW data pertaining to the $n=6,7$ levels not included in Liang \& Badnell (2010) have been retained for the present ion model. 


\subsection{Mg-like Ions}

\subsubsection{Ni XVII}

The data for Ni XVII have been replaced with the calculations published by Bhatia \& Landi (2011a). Their atomic model includes all 23 configurations with principal quantum number $n=3,4$ as well as $3 s 5 l$ with $l=s, p, d, f, g$, corresponding to 159 fine-structure levels, all of which have been included in CHIANTI. Bhatia \& Landi (2011a) reassessed all available laboratory and solar observations of Ni XVII lines and used them to remeasure the energy levels; the experimental energies they provide are taken from Feldman et al. (1971), Fawcett et al. (1972), Acton et al. (1985), Churilov et al. (1985), and Redfors (1988), as specified in the ELVLC file. A values and oscillator strengths were provided by Bhatia \& Landi (2011a) for all transitions in the atomic model. Collision strengths were calculated using the FAC code with the distorted wave approximation, at six values of the incident electron energy. The main limitation of this data set consists of the neglect of resonances; however, no data set has been provided so far that takes them into account with accuracy.

\subsection{Al-like Ions}

\subsubsection{Si II}

The CHIANTI model for Si II has been updated to include all fine-structure levels from the $3 s^{2} 3 p, 3 s 3 p^{2}, 3 s^{2} 3 d, 3 s^{2} 4 l$ $(l=s, p, d, f), 3 s^{2} 5 l(l=s, p, d)$, and $3 s^{2} 6 l(l=s, p)$ configurations, together with the $3 s 3 p\left({ }^{3} P\right) 3 d^{2} D_{J}$ levels, yielding 29 fine-structure levels in all. Effective collision strengths are taken from Tayal (2008a), who provided data calculated at 14 temperatures between $\log T=3.4$ and 5.4. The assessment of the collision data performed for CHIANTI revealed problems for 13 transitions whereby the collision strengths showed discontinuous jumps at low temperatures. The source of the problem was found to be errors in the threshold energies when computing the effective collision strengths, and so corrected data were provided to the CHIANTI team (S. S. Tayal 2009, private communication). Spline fits were performed to all transitions excited from the seven lowest energy levels, and these reproduce the original data to better than $1 \%$ for the full temperature range.

Radiative data are taken from Tayal (2007), supplemented with additional data for allowed transitions from Nahar (1998), and the ground forbidden transition decay rate from Nussbaumer (1977). Experimental energy values are available for all levels and are taken from version 3 of NIST. For transitions common to both the Tayal (2007) and Nahar (1998) data sets, agreement is found to within $\pm 60 \%$, and for transitions with $A$ values greater than $10^{6} \mathrm{~s}^{-1}$ to within $12 \%$. Proton rates for the ground transition are retained from the previous CHIANTI model (Young et al. 2003).

\subsubsection{Fe XIV}

Two new large-scale R-Matrix calculations have been recently published by Tayal (2008b) and Liang et al. (2010), which improved on the earlier calculation by Storey et al. (2000) available in earlier versions of CHIANTI, in that they employed larger targets. Tayal (2008b) reported a larger, 135 level Breit-Pauli $n=3$ R-matrix calculation, where the electron-impact excitation cross sections for the dipoleforbidden $3 s^{2} 3 p^{2} P_{3 / 2}-3 s^{2} 3 p^{2} P_{1 / 2}$ transition ("green coronal line") showed better agreement with laboratory measurements.
The calculation was entirely ab initio without the inclusion of experimental energies.

The Liang et al. (2010) calculations instead adopted experimental energies, whenever available. Liang et al. (2010) included in the close-coupling expansion the 197 lowest-lying levels of the 16 spectroscopically important $n=3,4$ configurations, plus additional CI with a further 77 configurations, for a total of 1241 LS terms and 2985 fine-structure levels. Liang et al. compared their energies, oscillator strengths, and collision strengths with those calculated by Storey et al. (2000) and Tayal (2008b), finding overall good agreement (to within 30\% for the rates of the strongest lines). Very good agreement in terms of line intensities with the results of the Storey et al. (2000) calculation at coronal temperatures was found by Liang et al.. The Liang et al. predicted intensities compare very well with laboratory and solar observations, as shown by Liang et al. (2010) and Del Zanna (2011c). The Liang et al. data were therefore adopted to replace the Storey et al. (2000) data. We fitted the Liang et al. (2010) effective collision strengths with nine-point splines; however, the structure of the data was such that only the data between $10^{5} \mathrm{~K}$ and $10^{7} \mathrm{~K}$ could be fitted. The $A$ values as calculated by Liang et al. (2010) were also adopted. Observed energies have been modified following a reassessment of the original wavelength measurements. The wavelengths from Churilov \& Levashov (1993) were adopted for most transitions, with the remaining from Shirai et al. (2000).

\subsubsection{Ni XVI}

The previous versions of CHIANTI had only a few observed energies listed for Ni XVI despite the fact that there have been many observations available in the literature. We have retained the theoretical energy levels from Bhatia \& Doschek (1999) and have replaced the experimental energies with the values from Fawcett \& Hatter (1980), Träbert et al. (1988), Redfors \& Litzen (1989), Hinnov et al. (1990), and Sugar et al. (1992). Most of the 40 levels have now been assigned an observed energy. Details on which level energy is associated with which reference are provided as comments within the ELVLC file.

\subsection{Si-like Ions}

\subsubsection{Fe XIII}

The previous Fe XIII model included electron-impact excitation rates from Gupta \& Tayal (1998), and $A$ values calculated for CHIANTI by Young (2004). They have been replaced with the new scattering calculation by Storey \& Zeippen (2010). The target included terms arising from the $3 s^{2} 3 p^{2}, 3 s 3 p^{3}, 3 s^{2} 3 p 3 d$, $3 p^{4}, 3 s 3 p^{2} 3 d, 3 s^{2} 3 d^{2}$, and $3 p^{3} 3 d$ configurations for a total of 114 fine-structure levels. For the lowest 27 levels, we adopt the Storey \& Zeippen (2010) A values, calculated using a large benchmark calculation, the term energy correction (TEC) procedure, and observed energies (see their Table 8). The rest of the $A$ values have been calculated by Del Zanna (2011b) using the same target as in the Storey \& Zeippen (2010) scattering calculation. The ordering of a few levels was modified to follow the ordering of the observed energies. For the observed energies, we adopt the values assessed by Del Zanna (2011b), who also identified a few new EUV lines, although some of them only tentatively.

The previous R-matrix calculations of Gupta \& Tayal (1998) and Aggarwal \& Keenan (2005) were found to have some shortcomings by Storey \& Zeippen (2010). The benchmark study by Del Zanna (2011b) found significant disagreements 
between predicted and observed line intensities for many lines using the Aggarwal \& Keenan (2005) data. A few problems with the Gupta \& Tayal (1998) data were also found. Very good overall agreement was found using the Storey \& Zeippen (2010) data. We have fitted the Storey \& Zeippen (2010) collision data with nine-point splines. The fits were accurate (to within $\approx 1 \%$ ) in the range $5.0 \leqslant \log T \leqslant 7.0$.

\subsection{2. $\mathrm{NiXV}$}

Ni XV collision excitation rates in the previous version of CHIANTI had not been changed since version 1 , and consisted of distorted wave calculations carried out using the UCL (University College London) suite of codes (Eissner \& Seaton 1972) with an atomic model that included the four lowest configurations; radiative data also came from an unpublished calculation carried out with SUPERSTRUCTURE using a 35 configuration atomic model. In the present version of CHIANTI, we have replaced the entire $\mathrm{Ni}$ XV data set with the calculation carried out by Landi \& Bhatia (2011).

The Ni Xv model adopted in CHIANTI includes nine configurations, for a total of 126 fine-structure levels: $3 s^{2} 3 p^{2}, 3 s 3 p^{3}$, $3 s^{2} 3 p 3 d, 3 p^{4}, 3 s 3 p^{2} 3 d$, and $3 s^{2} 3 p 4 l$, with $l=s, p, d, f$. The observed energy levels were remeasured by Landi \& Bhatia (2011) using all available laboratory and solar spectra, and we use the Landi \& Bhatia values in CHIANTI. These come from a variety of sources, depending on the configuration considered. The levels in the ground ${ }^{3} P$ multiplet are taken from the optical measurements of Jefferies et al. (1971) and Magnant Crifo (1973), the ground ${ }^{1} D$ level energy is determined from Curdt et al. (2004) and the energy of the ground ${ }^{1} S$ level comes from Sandlin et al. (1977). The energies of the other $n=3$ configurations come from combinations of energies from Fawcett \& Hayes (1972), Behring et al. (1976), Fawcett \& Hatter (1980), Kelly (1987), Träbert (1986, 1998), Träbert et al. (1988), Sugar et al. (1992), and Trigueiros et al. (2006). Energies for the $n=4$ configurations are taken from Fawcett et al. (1972) and Kastner et al. (1978).

The calculations of Landi \& Bhatia (2011) were carried out in the distorted wave approximation using the FAC ( $\mathrm{Gu} 2003$ ) on an atomic model that included 24 configurations: the entire $n=3$ complex, and the configurations $3 s^{2} 3 p 4 l, 3 s 3 p^{2} 4 l$, and $3 p^{3} 4 l$, with $l=s, p, d, f$. Landi \& Bhatia (2011) only provided data for the nine configurations that we included in CHIANTI. Radiative transition rates were retained for all the transitions involving the 126 levels in the CHIANTI Ni XV model, while only the collision excitation rates including levels with at least $1 \%$ fractional population at $\log N_{e}=12.0 \mathrm{~cm}^{-3}$ were retained; these were the ground configuration levels and the metastable $3 s^{2} 3 p 3 d^{3} F_{4}$ level. The main limitation of the Landi \& Bhatia (2011) data lies in the neglect of resonances, which can have a significant impact on the predicted emissivities. We are not aware of any calculation that included them.

\subsection{P-like Ions}

\subsection{1. $C l_{\text {III }}$}

Cl III is a new addition to the database and the model comprises the five levels of the ground configuration, $3 s^{2} 3 p^{3}$. Effective collision strengths are from Ramsbottom et al. (2001), and the CHIANTI spline fits are accurate to $0.56 \%$ or better over the temperature range 7500-250,000 K. Ramsbottom et al. (2001) did not provide radiative decay rates and so these are taken from Mendoza \& Zeippen (1982). Observed energies are from version 4 of the NIST database (Ralchenko et al. 2010).

\subsection{2. $K V$}

Some typographical errors were identified in the $\mathrm{K} v$ radiative data file (introduced in version 5) which have now been corrected. The only significant error was for the ${ }^{4} S_{3 / 2}-{ }^{2} P_{3 / 2}$ transition which had been inadvertently assigned the decay rate for the ${ }^{2} D_{5 / 2}-^{2} P_{1 / 2}$ transition. All other data remain the same.

\subsection{3. $\mathrm{Ca}$ VI}

Ca VI was not previously included in CHIANTI as no electron collision strengths were available for the ion. The first calculation was recently performed (Hudson 2009) and effective collision strengths for transitions between 39 fine-structure levels of the $3 s^{2} 3 p^{3}, 3 s 3 p^{4}$, and $3 s^{2} 3 p^{2} 3 d$ configurations of Ca VI were published. Unfortunately, all of these levels cannot be included in the CHIANTI model as fine-structure oscillator strengths or radiative decay rates are not available in the literature for all of these levels. In fact, the only oscillator strengths for Ca vi were published by Fawcett (1986), but this work did not include data for the intercombination transitions that are important for depopulating the ${ }^{4} D$ and ${ }^{4} F$ levels of the $3 s^{2} 3 p^{2} 3 d$ configuration. For this reason we have restricted the Ca VI model to only the five levels of the ground $3 s^{2} 3 p^{3}$ configuration for which we have used the radiative decay rates from Mendoza \& Zeippen (1982). The effective collision strengths between the ground levels are tabulated for the temperature range $4.5 \leqslant \log T \leqslant 6.5$, and were fit with nine-point splines accurate to $\leqslant 0.30 \%$. Energy levels are from version 3 of the NIST database.

\subsection{S-like Ions$$
\text { 2.13.1. Fe XI }
$$

A new R-matrix scattering calculation for electron collisional excitation of Fe XI was performed by Del Zanna et al. (2010). The target included the entire $n=3$ complex. The scattering calculation included 13 electron configurations giving rise to 465 LS terms and 1103 fine-structure levels. For the scattering calculation only the lowest 145 LS terms were retained, giving rise to 365 fine-structure levels. The ICFT method was used. Experimental energies were used within the calculation to accurately position the resonance thresholds. The radiative data were obtained with AUTOSTRUCTURE and adopted observed energies, whenever available. The new data allowed Del Zanna (2010) to provide the identifications and experimental energies of most of the $3 s^{2} 3 p^{3} 3 d$ levels. The benchmark study by Del Zanna (2010) showed good agreement between predicted and observed intensities. Comparisons with previous calculations have been presented in Del Zanna et al. (2010), where significant problems in all previous calculations were found. This comparison included the R-matrix calculations from Aggarwal \& Keenan (2003a, 2003b) and Gupta \& Tayal (1999a, 1999 b), as well as the distorted-wave ones from Bhatia \& Doschek (1996), which were included in the previous CHIANTI model.

\subsection{Ar-like Ions \\ 2.14.1. Fe IX}

Landi \& Young (2009a) identified in solar spectra observed by $S O H O / S U M E R$ the two Fe IX transitions $3 p^{5} 4 s^{3} P_{1}-3 p^{5} 4 p^{1} S_{0}$ 
and $3 p^{5} 4 s^{1} P_{1}-3 p^{5} 4 p{ }^{1} S_{0}$ at 717.661 and $803.422 \AA$, respectively. Combining with the energy of the $3 p^{5} 4 p{ }^{1} S_{0}$ level obtained by Young (2009), these wavelengths yield improved energies for the $3 p^{5} 4 s^{3} P_{1},{ }^{1} P_{1}$ levels of $950607 \pm 21$ and $965481 \pm$ $19 \mathrm{~cm}^{-1}$, respectively. These values have been added to the CHIANTI ELVLC file and used to generate new wavelengths in the WGFA file.

Landi \& Young (2009a) also carried out extensive calculations of $A$ values for all the configurations in the Fe IX atomic model in version 6 of CHIANTI, taken from Storey et al. (2002), using a very large atomic model and the FAC. They found that the Storey et al. (2002) A values were in excellent agreement with FAC results for all the $n=3$ configurations; on the contrary, the values for the $3 p^{5} 4 s$ and $4 p$ configurations needed improvement, due to the small number of $n=4$ configurations in the Storey et al. (2002) atomic model. Here we adopt the $A$ values from the "FAC-7" calculation of Landi \& Young (2009a) for the $4 s$ and $4 p$ configurations and retain the Storey et al. (2002) values already available in CHIANTI for the $n=3$ configurations.

\subsubsection{Ni XI}

The CHIANTI atomic model for Ni XI has been expanded to include the $3 s^{2} 3 p^{4} 3 d^{2}, 3 s^{2} 3 p^{5} 4 l$, and $3 s 3 p^{6} 4 l$ configurations, bringing the total amount of fine-structures levels to 180. These additional levels allow to predict a number of lines in the 120-200 $\AA$ wavelength range, although no observed energy levels were found for these additional configurations. The Edlén \& Smitt (1978) energies for the $3 s^{2} 3 p^{5} 3 d$ configurations have been retained. Theoretical energy levels, $A$ values, and collision strengths come from the calculations of Bhatia \& Landi (2011b) for all possible transitions between the 180 levels of the CHIANTI atomic model.

Bhatia \& Landi (2011b) provided distorted wave collision strengths calculated at six values of the incident electron energy, the lowest of which was very close to the threshold. The Bhatia \& Landi (2011b) calculations did not include resonant electron excitation; in order to include it for the lowest three configurations (lowest 17 fine-structure levels) the set of R-Matrix effective collision strengths of Aggarwal \& Keenan (2007) available in the previous version of CHIANTI was retained.

\subsection{K-like Ions}

\subsubsection{Ca II}

Effective collision strengths and radiative decay rates for 41 fine-structure levels belonging to the $3 p^{6} 3 d, 3 p^{6} 4 l(l=$ $s, p, d, f), 3 p^{6} 5 l(l=s, p, d, f, g), 3 p^{6} 6 l(l=s, p, d, f, g)$, $3 p^{6} 7 l(l=s, d, f, g)$, and $3 p^{6} 8 l(l=s, d, f, g)$ configurations have been calculated by Meléndez et al. (2007). Initial fits performed to the allowed transition collision strengths demonstrated that they were not tending to the high temperature limits, and so M. Meléndez recomputed the collision strengths and provided them to the CHIANTI team (M. Meléndez 2009, private communication). The revised data are in better agreement with the high temperature limits.

The effective collision strengths of the revised data set were tabulated over 16 temperatures from $3 \times 10^{3}$ to $4 \times 10^{4} \mathrm{~K}$, and spline fits were performed by the CHIANTI team to all transitions excited from the five lowest energy levels: $4 s^{2} S_{1 / 2}$, $3 d^{2} D_{3 / 2,5 / 2}$, and $4 p^{2} P_{1 / 2,3 / 2}$. The fits reproduce the original data to $\leqslant 0.76 \%$. Radiative decay rates are taken from Meléndez et al. (2007) who demonstrated good agreement with earlier calculations. Experimental energies are available for all 41 levels and are taken from version 3 of the NIST Atomic Database.

\subsubsection{Fe VIII}

Griffin et al. (2000) performed the calculation for Fe VIII available in the previous version of CHIANTI. It was a fairly large ICFT R-Matrix close-coupling calculation that included the 33 terms and the 77 levels of the configurations $3 s^{2} 3 p^{6} 3 d$, $3 s^{2} 3 p^{5} 3 d^{2}, 3 s^{2} 3 p^{5} 3 d 4 s, 3 s^{2} 3 p^{6} 4 l(l=s, p, d, f)$ in the close-coupling expansion. The Griffin et al. (2000) data have been benchmarked by Del Zanna (2009a) and Young \& Landi (2009), who showed that significant discrepancies (60\% or so) between Hinode/EIS observed intensities and those predicted with the Griffin et al. (2000) data exist. The discrepancies were explained by Del Zanna (2009a) with the run of a large-scale AUTOSTRUCTURE "benchmark" calculation. As already shown by Griffin et al. (2000), the introduction of additional configuration interactions changes significantly $(30 \%$ or so) the $A$ values for many of the strongest transitions. The effects are subtle in particular for strong transitions arising from very mixed levels, for which accurate level energies are particularly difficult to obtain as the level mixing changes when increasing the target.

In order to improve the Griffin et al. (2000) data, the collision strengths $\Omega$ of the dipole-allowed transitions were scaled with the ratio of the oscillator strengths $f$ and the energy differences $\Delta E$ (see, e.g., Flower \& Nussbaumer 1974):

$$
\Omega^{\prime}=\frac{\Delta E}{\Delta E^{\prime}} \frac{f^{\prime}}{f} \Omega,
$$

where $f^{\prime}$ and $\Delta E^{\prime}$ are the values calculated with the "benchmark" calculation. Improvement in the comparison with the Hinode/EIS data was found (Del Zanna 2009a). These scaled collision strengths were fitted with nine-point splines. For the radiative data, we adopt the AUTOSTRUCTURE results of the "benchmark" close-coupling calculation, with term energy corrections and observed energies, whenever available. For the observed energies, we adopt values assessed by Del Zanna (2009b) for the $3 s^{2} 3 p^{5} 3 d^{2}{ }^{4} D_{J}$ levels, and the $3 s^{2} 3 p^{6} 4 s$ level from Landi \& Young (2010).

\subsection{Ca-like Ions}

\subsubsection{Fe VII}

Fe VII was part of the original CHIANTI database release (Dere et al. 1997) and was updated in version 5 (Landi et al. 2006) with the data of Berrington et al. (2000). In each case, the model only consisted of the nine levels of the ground $3 p^{2} 3 d^{2}$ configuration. Zeng et al. (2005) and Witthoeft \& Badnell (2008) provided new atomic data (calculated with the distorted wave FAC code and with the R-matrix ones, respectively) for many additional levels, including those of the $3 p 3 d^{3}$ configuration, which gives rise to many lines at EUV wavelengths. Witthoeft \& Badnell (2008) found very good agreement (to within $\approx 20 \%$ ) between their effective collision strengths (and $A$ values) and those calculated by Zeng et al. at high temperatures (5.8 MK), but larger discrepancies (still within $40 \%$ ) at very low values (0.116 MK). Del Zanna (2009b) and Young \& Landi (2009) independently constructed Fe VII models using the Witthoeft \& Badnell (2008) atomic data and compared predicted line intensities with those observed 
by Hinode/EIS. Large discrepancies (factors of two) were found. Different conclusions were reached by the authors, particularly with regard line identifications, which demonstrates the complexity of the Fe VII model and the observed spectrum. Further work is required to resolve the discrepancies and so a new Fe vII model will not be released with CHIANTI 7.

\subsection{Ti-like Ions}

\subsection{1. $\mathrm{Fe} \mathrm{V}$}

Fe $\mathrm{v}$ is a new addition to CHIANTI and the model consists of all 37 levels of the ground $3 d^{4}$ configuration. Effective collision strengths for all transitions among the levels are taken from Ballance et al. (2007) who calculated values at 19 temperatures between $5 \times 10^{3}$ and $5 \times 10^{5} \mathrm{~K}$. The spline fits we made to the data are accurate to $0.59 \%$ or better over this range.

Ballance et al. (2007) did not give radiative decay rates for the forbidden transitions between the $3 d^{4}$ configuration levels and so values were taken from Nahar et al. (2000). Observed energies, which are available for all 37 levels, are from Ekberg (1975).

\subsection{V-like Ions}

\subsubsection{Fe IV}

A 37 level model of Fe IV has been constructed for CHIANTI containing all fine-structure levels of the ground $3 d^{5}$ configuration. Effective collision strengths are taken from Zhang \& Pradhan (1997) who tabulated values between 2000 and 500,000 K. Nine-point spline fits were performed by the CHIANTI team to each transition and these are accurate to $\leqslant 1.1 \%$ at all temperatures. Radiative data are from Froese Fischer et al. (2008), and observed energy levels are from version 3 of the NIST database.

Zhang \& Pradhan (1997) provided additional effective collision strengths for 103 levels of the $3 d^{4} 4 s$ and $3 d^{4} 4 p$, but these levels are not astrophysically important and so are not included in the CHIANTI model. We also note that Fe IV effective collision strengths have been presented by McLaughlin et al. (2005) and McLaughlin et al. (2006), however these are calculated in LS coupling and so are not suitable for CHIANTI. We note that McLaughlin et al. (2005) found differences of up to a factor two between their effective collision strengths and those of Zhang \& Pradhan when the latter values were averaged over the fine-structure levels. This suggests that a large fine-structure calculation for Fe IV would lead to significant changes to the Zhang \& Pradhan effective collision strengths.

\subsection{Mn-like Ions}

\subsubsection{Fe II}

The CHIANTI model of Fe II has remained unchanged since version 1 (Dere et al. 1997) and there has been a discrepancy between the wavelengths contained in the WGFA file and the energies in the ELVLC. For CHIANTI 7 the energies (which are from the NIST database) have been used to re-compute the Fe II wavelengths which replace the wavelengths in the WGFA file.

In addition, the radiative decay rate and oscillator strength for the c ${ }^{4} F_{7 / 2}-$ W ${ }^{4} G_{9 / 2}$ transition at $2509.097 \AA$ have been replaced with the values of Corrégé \& Hibbert (2005).

\subsection{Co-like Ions}

\subsubsection{Ni II}

Ni II is a new addition to CHIANTI and the atomic model consists of 17 levels from the $3 d^{9}$ and $3 d^{8} 4 s$ configurations.
Effective collision strengths are from the ICFT calculation of Bautista (2004) and are tabulated for 10 temperatures between 2000 and 30,000 K. Bautista (2004) provided data for 76 levels belonging to the $3 d^{9}, 3 d^{8} 4 s, 3 d^{7} 4 s^{2}, 3 d^{8} 4 p$ configurations. However, while fitting the data, some problems were found for transitions from the latter two configurations. For example, collision strengths for all transitions involving $3 d^{8} 4 s{ }^{2} \mathrm{H}_{11 / 2}$ were zero, and a number of the allowed transitions from $3 d^{8} 4 p$ did not tend toward their high temperature limit points. For this reason the CHIANTI model was restricted to the two lowest energy configurations. We also discounted the $3 d^{8} 4 s^{2} S_{1 / 2}$ level which has a significantly higher energy than all other levels in $3 d^{8} 4 s$, placing it above a number of the $3 d^{7} 4 s^{2}$ and $3 d^{8} 4 p$ levels. Therefore, decays to these levels are significant in de-populating $3 d^{8} 4 s^{2} S_{1 / 2}$, and so its population cannot be accurately modeled with a two configuration atomic model.

All transitions among the 17 levels were fit with nine-point splines by the CHIANTI team, and the effective collision strengths are reproduced to an accuracy of $\leqslant 0.6 \%$ over the entire temperature range. Experimental energy values were obtained for all levels from version 3 of the NIST atomic database. Bautista (2004) did not give radiative decay rates and so these are taken from Quinet \& Le Dourneuf (1996), supplemented with seven additional decay rates from Nussbaumer \& Storey (1982). Note that Nussbaumer \& Storey (1982) and Quinet \& Le Dourneuf (1996) assign the labels "2 $D_{5 / 2}$ " and " ${ }^{4} P_{5 / 2}$ " to the levels with energies $23,108.28 \mathrm{~cm}^{-1}$ and $25,036.38 \mathrm{~cm}^{-1}$, whereas the current version of the NIST database switches them: this happens because these levels are heavily mixed, so that the LS labels associated with them are somewhat arbitrary and can change in different calculations. Care was thus taken to ensure the level indices were correct in the CHIANTI WGFA file.

\section{SOFTWARE}

\subsection{ChiantiPy}

ChiantiPy is an implementation of the functionality of the CHIANTI IDL package in the Python programming language (http://python.org). Python is a modern, dynamic programming language, and its advantages include strong introspection capabilities and intuitive object orientation. ChiantiPy can be run in interactive command-line sessions within either the native Python or IPython environments. IPython (http://ipython.org) should be highly preferred, as it provides a powerful, interactive Python shell. If invoked as

$>$ ipython -pylab

the Python Matplotlib plotting library will be automatically loaded. ChiantiPy has been constructed as an object-oriented package that provide several main classes for the user.

ion: provides the basic functionality for individual ions in the CHIANTI database. This class enables calculations of level populations, spectral line emissivities, continua and spectra as a function of temperature and density.

continuum: enables the calculation of the free-free, free-bound, and two-photon continuum for a collection of ions in the database. The entire database can be used or selected ions.

spectrum: calculates the continuum and line spectrum over a specified wavelength range and applies a line profile. The line profile can be one of a box-car, a Gaussian with fixed spectral width, a Gaussian with a fixed resolving power $(\lambda / \delta \lambda)$, a Lorentz filter or a Moffat profile that is suitable for comparing with Chandra/LETG spectra. Other filters can be easily programmed by the user. 
mspectrum: allows the use of multiple cpu cores in the calculation of synthetic spectra that can be time consuming. It provides same functionality as spectrum and is invoked in the same way with the addition of the proc keyword that specifies the number of processors to use.

radLoss: calculates the optically thin radiative losses as a function of temperature and density.

The ChiantiPy package can be downloaded from http://source forge.net/projects/chiantipy/.

Documentation and instructions can be found at http://chian tipy.sourceforge.net/.

It is our intention to continue to maintain both the CHIANTI IDL and ChiantiPy packages at roughly the same level of functionality. The manner in which the two sets of programs are used is different: ChiantiPy is used by means of objects that have a number of advantages over the functional programming of the IDL package.

\section{SUMMARY}

In this paper, we have summarized the new features of version 7 of the CHIANTI database. They consist of data for several new ions, expanded and improved data for ions already in the database, and a new suite of programs (ChiantiPy) written in the Python computer language. CHIANTI 7 is a significant improvement over the previous databases and it allows a more complete and accurate calculation of the synthetic spectrum and of key lines routinely observed by the Hinode/EIS spectrometer.

The entire database as well as the standard suite of IDL computer programs are distributed through SolarSoft; they are also freely available at the CHIANTI Web site http://www.chiantidatabase.org. ChiantiPy can be found at http://chiantipy.sourceforge.net.

The work of E.L. is supported by the NNX10AM17G and NNX11AC20G NASA grants. G.D.Z. acknowledges support from STFC via the Advanced Fellowship program and the UK APAP Network. The work of P.R.Y. was performed under contract with the Naval Research Laboratory and was funded by NASA. K.P.D. acknowledges funding from the NASA APRA program. H.E.M. acknowledges support from STFC. The atomic data for MgIX, Si x, Fe VIII, Fe XI, Fe XIII, Fe XIV, and Fe XVII have been produced by the UK APAP Network, funded by the UK STFC under grant No. PP/E001254/1 with the University of Strathclyde. This research has made use of NASA's Astrophysics Data System. We warmly thank all the authors that provided us with the data necessary to make this version of the database possible, and all CHIANTI users that helped us improve the database and the software with their comments. We also thank the anonymous referee, whose comments helped us significantly clarify and improve this paper.

\section{REFERENCES}

Acton, L. W., Bruner, M. E., Brown, W. A., et al. 1985, ApJ, 291, 865 Aggarwal, K. M., Hamada, K., Igarashi, A., et al. 2008, A\&A, 487, 388 Aggarwal, K. M., \& Keenan, F. P. 2003a, A\&A, 399, 799

Aggarwal, K. M., \& Keenan, F. P. 2003b, MNRAS, 338, 412

Aggarwal, K. M., \& Keenan, F. P. 2005, A\&A, 441, 831

Aggarwal, K. M., \& Keenan, F. P. 2007, A\&A, 475, 393

Aggarwal, K. M., Keenan, F. P., \& Heeter, R. F. 2009, Phys. Scr., 80, 045301

Badnell, N. R. 1997, J. Phys. B: At. Mol. Opt. Phys., 29, 1

Ballance, C. P., Griffin, D. C., \& McLaughlin, B. M. 2007, J. Phys. B: At. Mol. Opt. Phys., 40, F327

Bautista, M. A. 2004, A\&A, 420, 763

Behring, W. E., Cohen, L., Doschek, G. A., \& Feldman, U. 1976, ApJ, 203, 521
Bell, K. L., Berrington, K. A., \& Thomas, M. R. J. 1998, MNRAS, 293, L83 Berrington, K. A., Nakazaki, S., \& Norrington, P. H. 2000, A\&AS, 142, 313 Beyer, H. F., Folkmann, F., \& Schartner, K.-H. 1986, Z. Phys. D, 1, 65 Bhatia, A. K., \& Doschek, G. A. 1996, At. Data Nucl. Data Tables, 64, 183 Bhatia, A. K., \& Doschek, G. A. 1999, At. Data Nucl. Data Tables, 71, 69 Bhatia, A. K., \& Landi, E. 2007, At. Data Nucl. Data Tables, 93, 742 Bhatia, A. K., \& Landi, E. 2011a, At. Data Nucl. Data Tables, 97, 189 Bhatia, A. K., \& Landi, E. 2011b, At. Data Nucl. Data Tables, 97, 50 Briand, J. P., Mosse, J. P., Indelicato, P., et al. 1983, Phys. Rev. A, 28, 1413 Brown, G. V., Beiersdorfer, P., Liedahl, D. A., Widmann, K., \& Kahn, S. M. 1998, ApJ, 502, 1015

Chen, M. H., Cheng, K. T., \& Johnson, W. R. 1993, Phys. Rev. A, 47, 3692

Churilov, S. S., Kononov, E. Ya., Ryabtsev, A. N., \& Zayikin, Yu. F. 1985, Phys. Scr., 32, 501

Churilov, S. S., \& Levashov, V. E. 1993, Phys. Scr., 48, 425

Corrégé, G., \& Hibbert, A. 2005, ApJ, 627, L157

Curdt, W., Landi, E., \& Feldman, U. 2004, A\&A, 427, 1045

Curdt, W., Landi, E., Wilhelm, K., \& Feldman, U. 2000, Phys. Rev. A, 62, 022502

Del Zanna, G. 2009a, A\&A, 508, 513

Del Zanna, G. 2009b, A\&A, 508, 501

Del Zanna, G. 2010, A\&A, 514, A41

Del Zanna, G. 2011a, A\&A, submitted

Del Zanna, G. 2011b, A\&A, 533, A12

Del Zanna, G. 2011c, A\&A, in press

Del Zanna, G., \& Ishikawa, Y. 2009, A\&A, 508, 1517

Del Zanna, G., Rozum, I., \& Badnell, N. R. 2008, A\&A, 487, 1203

Del Zanna, G., Storey, P. J., \& Mason, H. E. 2010, A\&A, 514, A40

Dere, K. P., Landi, E., Mason, H. E., Monsignori Fossi, B. C., \& Young, P. R. 1997, A\&AS, 125, 149

Dere, K. P., Young, P. R., Del Zanna, G., Landini, M., \& Mason, H. E. 2009, A\&A, 498, 915

Dohmann, H.-D., Liesen, D., \& Pfeng, H. 1978, Z. Phys. A, 285, 171

Dohmann, H.-D., \& Mann, R. 1979, Z. Phys. A, 291, 15

Dohmann, H.-D., \& Pfeng, H. 1978, Z. Phys, A, 288, 29

Drake, G. W. F. 1986, Phys. Rev. A, 34, 2871

Edlén, B. 1984, Phys. Scr., 30, 135

Edlén, B. 1985, Phys. Scr., 31, 345

Edlén, B., \& Smitt, R. 1978, Sol. Phys., 57, 329

Eissner, W., Jones, M., \& Nussbaumer, H. 1974, Comput. Phys. Commun., 8, 270

Eissner, W., \& Seaton, M. J. 1972, J. Phys. B: At. Mol. Phys., 5, 2187

Ekberg, J. O. 1975, Phys. Scr., 12, 42

Fawcett, B. C. 1970, J. Phys. B: At. Mol. Phys., 3, 1152

Fawcett, B. C. 1986, At. Data Nucl. Data Tables, 35, 203

Fawcett, B. C., Cowan, R. D., \& Hayes, R. W. 1972, J. Phys. B: At. Mol. Phys., 5,2143

Fawcett, B. C., \& Hatter, A. T. 1980, A\&A, 84, 78

Fawcett, B. C., \& Hayes, R. W. 1972, J. Phys. B: At. Mol. Phys., 5, 366

Feldman, U., \& Doschek, G. A. 2007, At. Data Nucl. Data Tables, 93, 779

Feldman, U., Katz, L., Behring, W., \& Cohen, L. 1971, J. Opt. Soc. Am., 61, 91

Ferner, E. 1941, Ark. Mat. Astron. Fysik, 28A, 1

Ferner, E. 1948, Ark. Mat. Astron. Fysik, 36A, 1

Flower, D. R., \& Nussbaumer, H. 1974, A\&A, 31, 353

Froese Fischer, C., Rubin, R. H., \& Rodríguez, M. 2008, MNRAS, 391, 1828

Froese Fischer, C., \& Tachiev, G. 2004, At. Data Nucl. Data Tables, 87, 1

Grant, I. P., McKenzie, B. J., Norrington, P. H., Mayers, D. F., \& Pyper, N. C. 1980, Comput. Phys. Commun., 21, 207

Griffin, D. C., Badnell, N. R., \& Pindzola, M. S. 1998, J. Phys. B: At. Mol. Opt. Phys., 31, 3713

Griffin, D. C., Pindzola, M. S., \& Badnell, N. R. 2000, A\&AS, 142, 217

Gu, M. F. 2003, ApJ, 582, 1241

Gupta, G. P., \& Tayal, S. S. 1998, ApJ, 506, 464

Gupta, G. P., \& Tayal, S. S. 1999a, ApJ, 510, 1078

Gupta, G. P., \& Tayal, S. S. 1999b, ApJS, 123, 295

Hinnov, E., Denne, B., Ramsey, A., Stratton, B., \& Timberlake, J. 1990, J. Opt. Soc. Am. B, 7, 2002

Hoory, S., Goldsmith, S., Feldman, U., Behring, W., \& Cohen, L. 1971, J. Opt. Soc. Am., 61, 504

Hudson, C. E. 2009, Phys. Scr., 79, 035301

Hutton, R., Restad, N., Engstroem, L., \& Huldt, S. 1985, Phys. Scr., 31, 506

Jefferies, J. T., Orrall, F. Q., \& Zirker, J. B. 1971, Sol. Phys., 16, 103

Kastner, S. O., Swartz, M., Bhatia, A. K., \& Lapides, J. 1978, J. Opt. Soc. Am., 68,1558

Keenan, F. P., Berrington, K. A., Burke, P. G., Dufton, P. L., \& Kingston, A. E. 1986, Phys. Scr., 34, 216

Kelly, R. L. 1987, J. Phys. Chem. Ref. Data, Vol. 16 
Kramida, A. E., Bastin, T., Biémont, E., Dumont, P.-D., \& Garnir, H.-P. 1999, Eur. Phys. J. D, 7, 525

Landi, E., \& Bhatia, A. K. 2011, At. Data Nucl. Data Tables, in press

Landi, E., Del Zanna, G., Young, P. R., et al. 2006, ApJS, 162, 261

Landi, E., \& Gu, M. F. 2006, ApJ, 640, 1171

Landi, E., Landini, M., Dere, K. P., Young, P. R., \& Mason, H. E. 1999, A\&AS, 135,339

Landi, E., \& Young, P. R. 2009a, ApJ, 707, 1191

Landi, E., \& Young, P. R. 2009b, ApJ, 706, 1

Landi, E., \& Young, P. R. 2010, ApJ, 713, 205

Liang, G. Y., \& Badnell, N. R. 2010, A\&A, 518, A64

Liang, G. Y., Badnell, N. R., López-Urrutia, C., et al. 2010, ApJS, 190, 322

Liang, G. Y., Whiteford, A. D., \& Badnell, 2009, A\&A, 499, 943

Loch, S. D., Pindzola, M. S., Ballance, C. P., \& Griffin, D. C. 2006, J. Phys. B, 39, 85

Magnant Crifo, F. 1973, Sol. Phys., 31, 91

Martin, W. C., Sugar, J., Musgrove, A., \& Dalton, G. R. 1995, NIST Database for Atomic Spectroscopy, Version 1.0, NIST Standard Reference Database 61

McLaughlin, B. M., Hibbert, A., Scott, M. P., et al. 2005, J. Phys. B: At. Mol. Opt. Phys., 38, 2029

McLaughlin, B. M., Hibbert, A., Scott, M. P., et al. 2006, A\&A, 446, 1185

Meléndez, M., Bautista, M. A., \& Badnell, N. R. 2007, A\&A, 469, 1203

Mendoza, C., \& Zeippen, C. J. 1982, MNRAS, 198, 127

Merkelis, G., Martinson, I., Kisielius, R., \& Vilkas, M. J. 1999, Phys. Scr., 59 , 122

Merkelis, G., Vilkas, M. J., Kisielius, R., \& Gaigalas, G. 1997, Phys. Scr., 56, 41

Nahar, S. N. 1998, At. Data Nucl. Data Tables, 68, 183

Nahar, S. N., Delahaye, F., Pradhan, A. K., \& Zeippen, C. J. 2000, A\&AS, 144, 141

Neill, P. A., Traebert, E., Beiersdorfer, P., et al. 2000, Phys. Scr., 62, 141

Neupert, W. M. 1971, Sol. Phys., 18, 474

Nussbaumer, H. 1977, A\&A, 58, 291

Nussbaumer, H., \& Storey, P. J. 1982, A\&A, 110, 295

Parpia, F. A., \& Johnson, W. R. 1982, Phys. Rev. A, 26, 1142

Penn, M. J., \& Kuhn, J. R. 1994, ApJ, 434, 807

Phillips, K. J. H., Sylwester, J., Sylwester, B., \& Landi, E. 2003, ApJ, 589, L113

Quinet, P., \& Le Dourneuf, M. 1996, A\&AS, 119, 99

Ralchenko, Yu., Kramida, A. E., \& Reader, J. NIST ASD Team 2010 NIST Atomic Spectra Database (version 4.0.1; Gaithersburg, MD: National Institute of Standards and Technology), Available online at http://physics.nist.gov/asd (2010 December 1)
Ralchenko, Yu., Kramida, A. E., Reader, J., et al. 2008, NIST Atomic Spectra Database (ver. 3.1.5; Gaithersburg, MD: National Institute of Standards and Technology)

Ramsbottom, C. A., Bell, K. L., \& Keenan, F. P. 1998, MNRAS, 293, 233

Ramsbottom, C. A., Bell, K. L., \& Keenan, F. P. 2001, At. Data Nucl. Data Tables, 77, 57

Redfors, A. 1988, Phys. Scr., 38, 702

Redfors, A., \& Litzen, U. 1989, J. Opt. Soc. Am. B, 6, 1447

Rice, J. E., Reinke, M. L., Ashbourn, J. M. A., et al. 2011, J. Phys. B: At. Mol. Opt. Phys., 44, 165702

Sandlin, G. D., Brueckner, G. E., \& Tousey, R. 1977, ApJ, 214, 898

Shirai, T., Sugar, J., Musgrove, A., \& Wiese, W. L. 2000, J. Phys. Chem. Ref. Data, Monograph 8

Storey, P. J., Mason, H. E., \& Young, P. R. 2000, A\&ASS, 141, 285

Storey, P. J., Zeippen, C. J., \& Le Dourneuf, M. 2002, A\&A, 394, 753

Storey, P. J., \& Zeippen, C. J. 2010, A\&A, 511, A78

Sugar, J., Kaufman, V., \& Rowan, W. L. 1992, J. Opt. Soc. Am. B, 9, 344

Sylwester, J., Sylwester, B., Culhane, J. L., et al. 2003, in Solar Variability as an Input to the Earth's Environment, ed. A. Wilson (ESA SP-535; Noordwijk: ESA), 733

Tayal, S. S. 2007, J. Phys. B: At. Mol. Opt. Phys., 40, 2551

Tayal, S. S. 2008a, ApJS, 179, 534

Tayal, S. S. 2008b, ApJS, 178, 359

Träbert, E. 1986, Z. Phys. D, 2, 213

Träbert, E. 1998, MNRAS, 297, 399

Träbert, E., Heckmann, P. H., Hutton, R., \& Martinson, I. 1988, J. Opt. Soc. Am. B, 5, 2173

Trigueiros, A. G., Borges, F. O., Cavalcanti, G. H., \& Farias, E. E. 2006, J. Quant. Spectrosc. Radiat. Transfer, 97, 29

Vilkas, M. J., Ishikawa, Y., \& Träbert, E. 2005, Phys. Scr., 72, 181

Walker, A. B. C., Jr., Rugge, H. R., \& Weiss, K. 1974, ApJ, 188, 423

Whiteford, A. D., Badnell, N. R., Ballance, C. P., et al. 2001, J. Phys. B: At Mol. Opt. Phys., 34, 3179

Wilhelm, K., Curdt, W., Marsch, E., et al. 1995, Sol. Phys., 162, 189

Witthoeft, M. C., \& Badnell, N. R. 2008, A\&A, 481, 543

Young, P. R. 2004, A\&A, 417, 785

Young, P. R. 2009, ApJ, 691, L77

Young, P. R., Del Zanna, G., Landi, E., et al. 2003, ApJS, 144, 135

Young, P. R., \& Landi, E. 2009, ApJ, 707, 173

Zatsarinny, O., \& Tayal, S. S. 2003, ApJS, 148, 575

Zeng, J., Liang, G. Y., Zhao, G., \& Shi, J. R. 2005, MNRAS, 357, 440

Zhang, H. L., \& Pradhan, A. K. 1997, A\&AS, 126, 373

Zhang, H. L., \& Sampson, D. H. 1987, ApJS, 63, 487 